\title{
Decrease of Glucose in the Human Visual Cortex during Photic Stimulation
}

\author{
Klaus-Dietmar Merboldt, Harald Bruhn, Wolfgang Hänicke, \\ Thomas Michaelis, and Jens Frahm
}

Max-Planck-Institut für biophysikalische Chemie, Postfach 2841, D-3400 Göttingen. Germany

Received December 9, 1991; revised February 25, 1992

\begin{abstract}
Localized proton NMR spectroscopy was used to study cerebral metabolism in the visual cortex of healthy adults during rest and photic stimulation. Basal lactate levels showed considerable interindividual differences ranging from below detectability $(<0.3$ $\mathrm{m} M$ ) to about $1 \mathrm{~m} M$ without consistent alterations during photic stimulation. Local brain glucose levels were significantly reduced $(\approx 50 \%)$ during the entire period of photic stimulation and recovered to resting levels $(\approx 0.8 \mathrm{mM})$ within $10 \mathrm{~min}$ after the end of stimulation. This decrease reflects the establishment of a new equilibrium due to enhanced delivery (blood flow) and enhanced consumption. The absence of lactate accumulation supports the hypothesis of a rapid efflux of lactate from brain tissue under activated conditions. (c) 1992 Academic Press, Inc.
\end{abstract}

Neural activity may be enhanced during physiologic stimulation in circumscribed regions of the brain. Its coupling to glucose utilization, blood flow, and oxygen extraction has been investigated using positron emission tomography, autoradiography, and bioluminescence (1-5). Here noninvasive, localized proton nuclear magnetic resonance (NMR) spectroscopy was used to determine lactate and glucose concentrations in the visual cortex of man during rest and photic stimulation.

\section{METHODS}

Proton NMR studies were carried out at a magnetic field strength of $2.0 \mathrm{~T}$ using a whole-body NMR imaging system (Siemens Magnetom). Spectra were obtained from volumes-of-interest ( VOI) in the occipital lobe covering the midsagittal visual cortex and subjacent white matter depending on the size of the VOI. In all cases the position and size of the VOI were defined by NMR imaging as shown in Fig. 1. Spectral localization was achieved by means of a stimulated echo acquisition mode (STEAM) radiofrequency (RF) pulse and gradient sequence as described previously (6). Since the method applies three slice-selective RF pulses in orthogonal directions with the VOI data given by the stimulated echo signal from the intersection of the excited slices, direct localization is performed within a single scan without the ambiguity of a multi-scan subtraction technique.

Binocular photic stimulation was accomplished by pulses of red light transmitted into the NMR magnet via glass fiber optics that were arranged in an equidistant matrix of $4 \times 5$ dots $\left(15 \times 15 \mathrm{~mm}^{2}\right)$ for each eye of the subjects $\left(50 \mathrm{~mm}\right.$ distance, $18^{\circ}$ view 




FIG. 1. Oblique brain section reconstructed from a three-dimensional $128 \times 256 \times 256 \mathrm{~T}$ 1-weighted NMR data set $\left(2.0 \mathrm{~T}\right.$ ) of a young adult ( $R F$ spoiled FLASH. TR $=15 \mathrm{~ms}, \mathrm{TE}=6 \mathrm{~ms}, 20^{\circ}$ flip angle, section thickness $1 \mathrm{~mm}$, measuring time $8 \mathrm{~min}$, imaging headcoil $)$. The box $\left(20 \times 20 \mathrm{~mm}^{2}\right)$ indicates the upper level of the typical position of an 8-ml volume-of-interest (VOI) selected for localized proton NMR spectroscopy in the visual cortex.

angle). Following an initial period of at least $30 \mathrm{~min}$ of dark adaptation in the magnet, proton NMR spectra with a measuring time of $6.5 \mathrm{~min}$ were acquired before, during, and after stimulation.

The examinations were carried out on young healthy adults ( $n=48,20-43$ years, mean 27.5 years). Written informed consent was obtained from all subjects prior to investigation. In the course of the study the frequency of stimulation $(4-16 \mathrm{~Hz})$, the size of the VOI $(8-64 \mathrm{ml})$, the echo time TE $(20-270 \mathrm{~ms})$ and the middle interval 
TM (30-60 ms) of the STEAM sequence, and the RF coils (imaging headcoil, combination of Helmholtz transmission coils with $10 \mathrm{~cm}$ diameter surface receive coil) were varied for two reasons: first, to match the protocol to that of previous NMR studies of lactate during photic stimulation (7-9) and, second, to optimize the detectability of glucose resonances with the use of short-echo time STEAM spectroscopy. Therefore, the glucose part of the study involved only a subgroup of volunteers $(n=$ $7,24-38$ years, mean 29.3 years) investigated under exactly the same experimental conditions ( $8 \mathrm{ml}$ VOI, TE $=20 \mathrm{~ms}, \mathrm{TM}=60 \mathrm{~ms}$, surface receive coil, $16 \mathrm{~Hz}$ photic stimulation, full time course including a recovery period after stimulation ). Statistical analysis of the glucose data was performed using a one-sided Student's $t$ test of paired differences (before/during stimulation of the same volunteer) of the integrated 3.43ppm glucose resonance signal.

\section{RESULTS}

In contrast to other metabolites such as $N$-acetylaspartate (NAA) or creatine and phosphocreatine $(\mathrm{Cr})$, lactate levels showed considerable interindividual variability in proton NMR spectra of normal human brain in vivo (10). Spectra of different subjects revealed resting lactate concentrations from below detectability $(<0.3 \mathrm{~m} M)$ to about $1 \mathrm{~m} M$ with predominantly low values. In further studies comprising additional volunteers no significant changes were observed during the course of a day, in parietal and occipital gray matter as well as in white matter, after extensive physical exercise, and after one subject fell asleep. Further evidence for a low mean cerebral lactate level comes from similar observations in more than 300 other subjects and 200 patients examined in this laboratory within the past 2 years.

The top trace in Fig. 2 shows a typical short-echo time proton NMR spectrum from an 8-ml VOI in the visual cortex of a resting subject (supine position, ear plugs, darkness). While the strongly coupled resonance of glucose (Glc) at $3.43 \mathrm{ppm}$ chemicalshift frequency $(11-15)$ is clearly visible, the spectrum does not show a doublet signal from lactate at $1.33 \mathrm{ppm}$. Under resting conditions the glucose concentration in the visual cortex was estimated to be about $0.8 \mathrm{~m} M$. This value as well as the lactate concentrations given above were derived relative to an average NAA concentration of approximately $10 \mathrm{~m} M$ in gray/white matter, e.g., see (16).

The time course of the lactate signal during photic stimulation exhibited all possible patterns including decreasing levels $(n=6)$, increasing levels $(n=10)$, and constant levels of about $0.5-1.0 \mathrm{~m} M(n=11)$ and $<0.3 \mathrm{~m} M(n=21)$. In contrast, cerebral glucose was consistently decreased in all cases where the spectroscopic conditions were appropriate for its observation $(n=7)$. In these experiments lactate was seen in two volunteers in only one or two spectra of the total protocol. As illustrated by a comparison of the two central spectra in Fig. 2 the decrease of the glucose signal was evident in the first 0 - to 7 -min spectrum acquired after the onset of stimulation. The bottom spectrum demonstrates recovery of the resting glucose level in the dark state 7-13 min after the end of stimulation.

Complementary to the direct observation of the 3.43-ppm signal reduction, Fig. 3 shows the data in the form of a difference spectrum (b) in comparison to a glucose spectrum obtained under identical conditions (a). The difference spectrum was obtained by subtracting the in vivo spectra before and during photic stimulation ( $n$ 
$=7$ ) with the phase and frequency of the creatine resonance taken as a reference. Although the 3.43-ppm resonance of glucose is clearly identified in the difference spectrum, the observation of the second glucose resonance at $3.80 \mathrm{ppm}$ is hampered by residual subtraction errors from overlapping and neighboring resonances (glutamate, myo-inositol, creatine/phosphocreatine). In Fig. 4 changes in the glucose concentrations were normalized to the individual basal levels. Each bar corresponds to a 6.5 min spectral acquisition. Obviously, the decreased cerebral glucose level persisted throughout the entire period of photic stimulation with an approximate twofold reduction as compared to basal concentrations (paired Student's $t$ test, $p<0.01$ ). Full recovery of the glucose concentration was observed $10-15 \mathrm{~min}$ after the end of stimulation.

\section{DISCUSSION}

The decrease of the local cerebral glucose concentration during photic stimulation may be understood as a balancing between enhanced glucose utilization and glucose delivery by stimulated blood flow. While the altered rates of consumption and supply establish a new equilibrium, very little direct information has been obtained about resulting steady-state tissue concentrations. In chloralose-anesthetized rats somatosensory stimulation has been reported to result in a $21 \%$ decrease of tissue glucose content (2). The approximate $50 \%$ reduction of the glucose level in the human visual cortex reported here is based on the observation that the 3.43-ppm resonance area is largely unaffected by other metabolites, e.g., taurine (12). This is supported by a comparison of in vivo and postmortem localized proton NMR spectra from rat brain. Although the taurine concentration in rat brain is about fourfold that of adult human brain (17), the 3.43-ppm resonance became rapidly and almost completely depleted after sacrificing the animal (11). Furthermore, transient undershoot hypoglycemia ( $2.5 \mathrm{~m} \mathrm{M}$ plasma glucose concentration) after a glucose infusion protocol in volunteers resulted in a decrease of the 3.43-ppm resonance similar to that under photic stimulation (unpublished data). Finally, glucose contributions from the cerebrospinal fluid ( $4 \mathrm{~m} M$ ) accounted for less than $0.2 \mathrm{~m} M$ as assessed by decreasing the lateral dimension of the VOI. Variations of this contribution were ruled out by the investigation of fasted subjects under controlled blood glucose levels.

The present findings contribute new evidence to the ongoing discussion about metabolic control of glucose utilization in the awake human brain. The variability of cerebral lactate levels as well as the absence of any consistent correlation between brain lactate and photic stimulation indicate that lactate levels do not necessarily provide a representative and direct measure of physiologic activity. Previous NMR

FIG. 2. Localized proton NMR spectra $(2.0 \mathrm{~T})$ from an $8-\mathrm{ml} \mathrm{VOI}$ in the visual cortex of a 24-year-old subject (STEAM localization sequence, TE $=20 \mathrm{~ms}, \mathrm{TM}=60 \mathrm{~ms}, \mathrm{TR}=3000 \mathrm{~ms}, 128 \mathrm{scans}, 10 \mathrm{~cm}$ diameter surface receive coil). Resonances are assigned to $N$-acetylaspartate (NAA), glutamate (Glu), creatine and phosphocreatine ( $\mathrm{Cr}$ ), choline-containing compounds (Cho), glucose (Glc), and myo-inositol (Ins). A doublet signal from the methyl group of lactate at $1.33 \mathrm{ppm}$ is not detected. The $\mathrm{Cho} / \mathrm{Cr}$ ratio is characteristic of gray matter. (a) Resting spectrum after 30 min of dark adaptation, (b) expanded section of spectrum (a) (2.9-3.9 ppm chemical-shift range, amplitude factor 4), (c) expanded section of a spectrum obtained during the first $6.5 \mathrm{~min}$ of $16 \mathrm{~Hz}$ photic stimulation, and (d) expanded section of a spectrum obtained $7-13 \mathrm{~min}$ after the end of photic stimulation. 

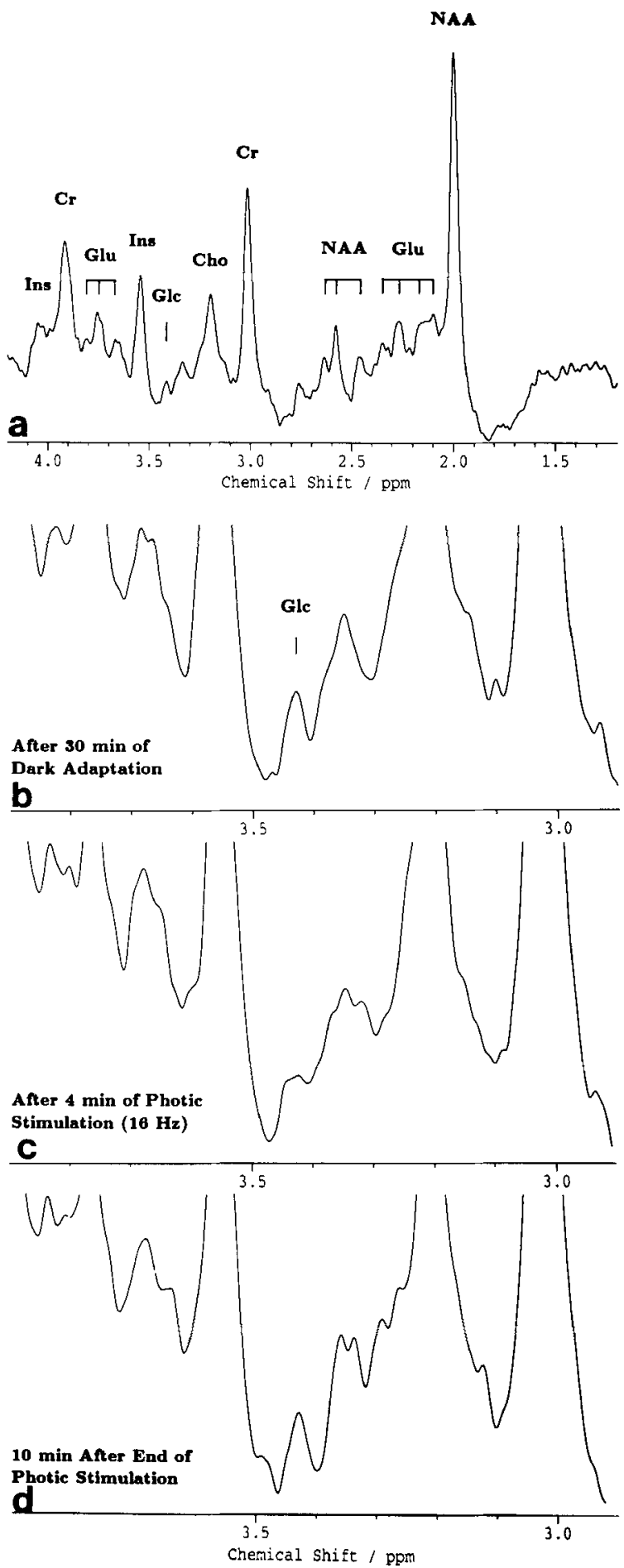

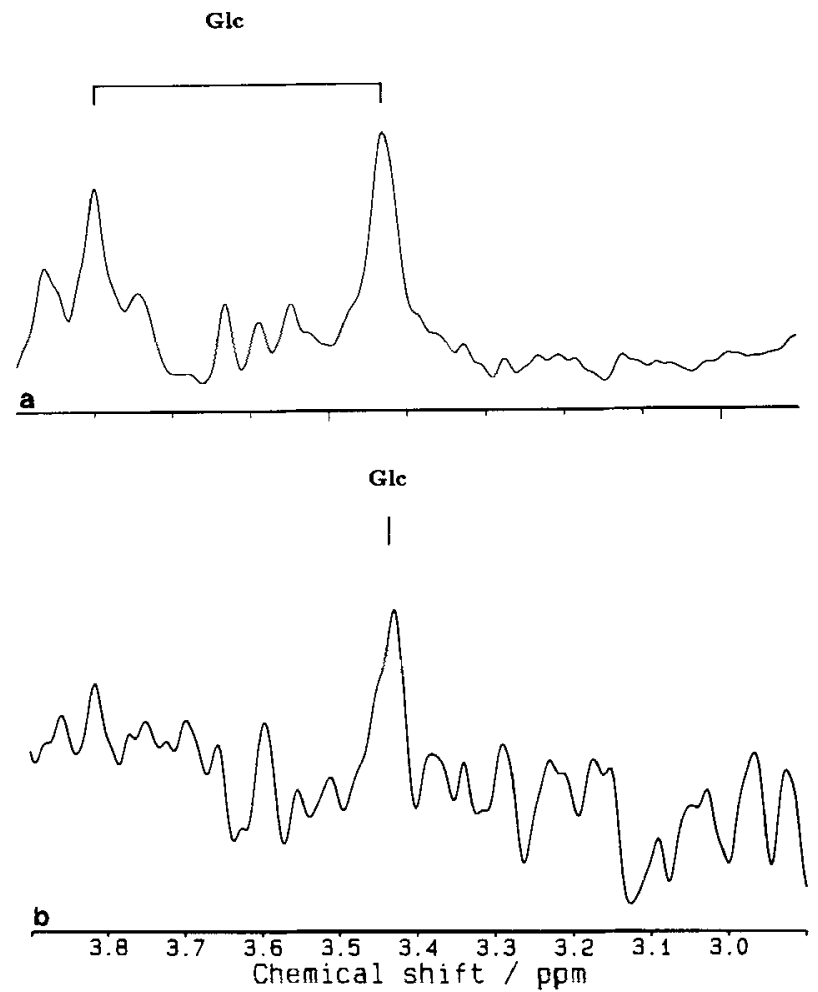

FIG. 3. (a) Localized proton NMR spectrum $(2.0-\mathrm{T}, \mathrm{TR}=6000 \mathrm{~ms}$, TE $=20 \mathrm{~ms})$ of an aqueous solution of D-glucose. Line-broadening was applied to mimic in vivo conditions. (b) Difference spectrum obtained by subtracting the in vivo spectra ( $n=7$ ) before and during $16 \mathrm{~Hz}$ photic stimulation (parameters as in Fig. 2).

findings of an increase of cerebral lactate in the human visual cortex upon photic stimulation (7-9) are not confirmed. While minor differences in the protocols cannot be excluded, corresponding effects on the metabolic response in the visual system are not likely unless different cell populations were selectively activated with various stimuli. This remains to be established.

The assumption that an excess demand of energy and a delayed supply of oxygen would cause glycolysis to switch to anaerobic production of lactate is not very likely in view of the small amount of energy needed for additional neuronal spike activity (18) and the immediacy of blood flow enhancement in the posterior cerebral artery under photic stimulation (19). The latter effect leads to an increased supply of oxygen but not to a corresponding increase in oxygen consumption ( 1 ) and channeling of glucose metabolites into oxidative respiration. Since our observations suggest an effective decoupling of enhanced glucose consumption and lactate accumulation, the hypothesis of increased "aerobic" glycolysis during stimulated activity remains questionable. However, if increased lactate production occurs without the accumulation of lactate, then the present data lend further support for the metabolic model of glucose utilization and blood flow (20) in which glucose consumption is followed by a rapid 


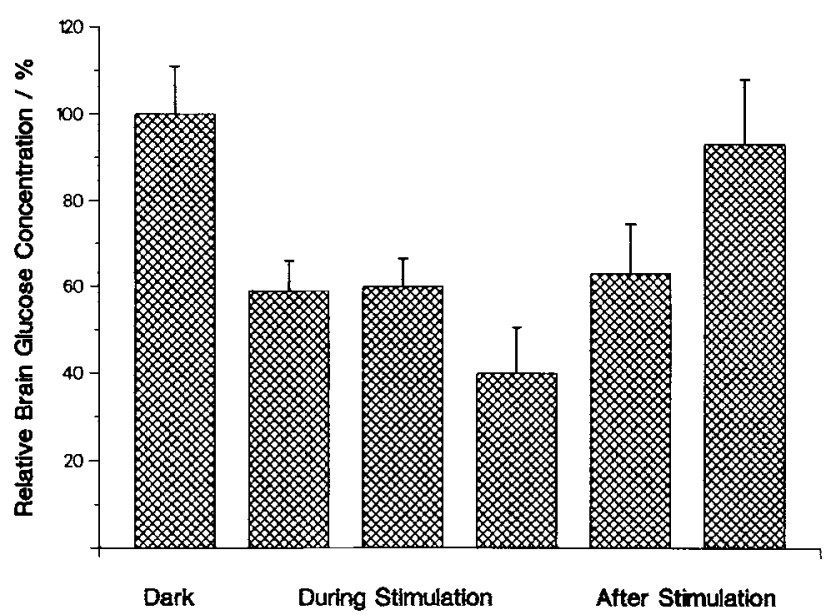

FIG. 4. Brain glucose levels in percentage of resting value ( \pm standard error of the mean, $n=7$ ) before, during, and after $16 \mathrm{~Hz}$ photic stimulation. The data were obtained by integration of the 3.43-ppm resonance entirely assigned to glucose. The bars represent successive $6.5 \mathrm{~min}$ spectral acquisitions. The decrease of glucose during stimulation is statistically significant (paired Student's $t$ test, $p<0.01$ ).

efflux of lactate from the tissue. In fact, lactate clearance has been shown to become particularly effective under activated conditions (2l).

\section{ACKNOWLEDGMENTS}

Financial support by the Bundesminister für Forschung und Technologie of the Federal Republic of Germany (Grant 01 VF 8606/6) is gratefully acknowledged. Figure 4 has been generated using the facilities of the Gesellschaft für wissenschaftliche Datenverarbeitung, Göttingen.

\section{REFERENCES}

1. P. T. Fox, M. E. Raichle, M. A. Mintun, and C. Dence, Science 241, 462 (1988).

2. M. Ueki, F. Linn, And K. A. Hossmann, J. Cereb. Blood Flow Metab. 8, 486 (1988).

3. R. Frackowiak, G. L. Lenzi. T. Jones, AND J. Heather, J. Comput. Assist. Tomogr. 4, 727 (1980).

4. M. Phelps. S. C. Huang, E. Hoffman, C. Selin, L. Sokoloff, and D. Kuhl. Ann. Neurol. 6, 371 (1979).

5. L. Sokoloff, M. Reivich, C. Kennedy, M. H. Des Rosiers, C. S. Patlak, K. D. Pettigrew, O. SAKURADA, AND M. SHINOHARA, J. Neurochem. 28, 897 (1977).

6. J. Frahm, T. Michaelis, K. D. Merboldt, H. Bruhn, M. L. Gyngell, and W. Hänicke, J. Magn. Reson. 90, 464 (1990).

7. J. W. PRICHARD, NMR Biomed. 4, 99 (1991).

8. J. W. Prichard, D. L. Rothman, E. Novotny, O. A. C. Petroff, T. Kuwabara, M. Avison, A. Howseman. C. C. Hanstock, and R. G. Shulman, Proc. Nall. Acad. Sci. USA 88, 5829 (1991).

9. D. Sappey-Marinier, G. Calabrese, J. Hugg, R. Deicken, G. Fein, and M. Weiner, "Book of Abstracts, Society of Magnetic Resonance in Medicine, Ninth Annual Meeting, August 18-24, 1990, New York," p. 106.

10. K. D. Merboldt, H. Bruhn, M. L. Gyngell, W. Hänicke, T. Michaelis, and J. Frahm, "Book of Abstracts. Society of Magnetic Resonance in Medicine, Tenth Annual Meeting, August 10-16, 1991, San Francisco," p. 392.

11. M. L. Gyngell, T. Michaelis, D. HörstermanN, H. Bruhn, W. Hänicke, K. D. MERboldt, and J. Frahm, Magn. Reson. Med. 19, 489 (1991). 
12. T. Michaelis, K. D. Merboldt, W. Hänicke, M. L. Gyngell, H. Bruhn, and J. Frahm, NMR Biomed. 4, 90 (1991).

13. R. Gruetter, D. L. Rothman, E. J. Novotny, G. I. Shulman, J. W. Prichard, and R. G. Shulman, "Works in Progress, Society of Magnetic Resonance in Medicine, Tenth Annual Meeting, August 10-16, 1991, San Francisco," p. 1014.

14. H. Bruhn, T. Michaelis, K. D. Merboldt, W. Hänicke, M. L. Gyngell, and J. Frahm, Lancet 337, 745 (1991).

15. R. KReIS AND B. D. Ross, "Book of Abstracts, Society of Magnetic Resonance in Medicine. Tenth Annual Meeting, August 10-16, 1991, San Francisco," p. 400.

16. T. Michaelis, H. Bruhn, M. L. Gyngell, W. Hänicke, K. D. Merboldt, and J. Frahm, "Book of Abstracts, Society of Magnetic Resonance in Medicine, Tenth Annual Meeting, August 10-16, 1991, San Francisco," p. 387.

17. T. L. Perry, S. Hansen, and S. S. Gandham, J. Neurochem. 36, 406 (1981).

18. O. D. Creutzfeldt, "Brain Work: The Coupling of Function, Metabolism, and Blood Flow in the Brain" (D. H. Ingvar and N. A. Lassen, Eds.), pp. 22-47, Alfred Benzon Symposium VIII, Munksgaard, Copenhagen, 1975.

19. R. AASLID, Stroke 18, 771-775 (1987).

20. R. F. ACKermanN AND J. L. LeAR, J. Cereb. Blood Flow Metab. 9, 774 (1989).

21. J. L. Lear AND R. K. Kasliwal, J. Cereb. Blood Flow Metab. 11, 576 (1991). 